\title{
Revisiting on the Problems of Crew Scheduling
}

\author{
Jooyoung Kim ${ }^{1}$, Sangchul Jung ${ }^{2}$ and Seungjae Lee ${ }^{3 *}$ \\ ${ }^{1}$ Integrated Urban Research Center, University of Seoul, South Korea \\ ${ }^{2}$ Department of Transportation Engineering, University of Seoul, South Korea \\ 3*Department of Transportation Engineering, University of Seoul, South Korea \\ ${ }^{1}$ trafficplan@naver.com, ${ }^{2}$ lich13@naver.com, ${ }^{3}$ sjlee@uos.ac.kr
}

\begin{abstract}
For the traditional crew scheduling problem, the main object was to reduce the number of crews and vehicles. However, there are much more things which need to be considered, such as the quality of crew schedule. The importance of improving the quality in crew schedule is not being considered well in current situation. Now, it is time to consider the problem in the other perspective.

In this paper, the general description of CSP is introduced. The main objective of CSP is to minimize the operation cost, by optimizing crew schedule. For this purpose, various studies were conducted in various situations. The models, the methodologies and the algorithms for solving CSP are presented by reviewing the previous works. For each methodology and algorithm, rough processes are suggested. Our study presents implication deducted by reviewing. Requirements for the future works are also presented.
\end{abstract}

Keywords: Crew Scheduling, Public Transportation, Optimization

\section{Introduction}

For most of public transportation company, the main issue is to minimize their operation cost. Because the paid cost to crews takes a large amount of entire cost, Crew Scheduling Problem arises (Valdes and Andres, 2010). Crew Scheduling Problem (CSP) is generally defined as creating a set of crew duties to serve the daily transport service by generating sequence of trips and assigning crews to them in minimum cost. However, it is hard to solve or unable to solve in some situations. Thus, researches have been conducting to find fast and efficient ways to obtain the optimal solution of crew scheduling problem in many situations.

For optimizing crew schedule, it is necessary to model CSP. By representing CSP into mathematical formulation, it is possible to apply optimizing processes. In general, CSP is modelled to Set Covering problem, Set Partitioning problem, Bin Packing problem, etc. In process of modeling CSP, some problems rose such as the large size of CSP or becoming complex problem. Naturally, it is hard to solve CSP because of its attribute that its models are NP-hard. Even notwithstanding this problem, the size of CSP becomes large for reflecting real-world to the model. In addition, the formulated form of CSP is being complex by the result of continuous research with considering various constraints. Thus, models for representing CSP are being more sophisticated and complex simultaneously.

These problems, the large size and the complexity of CSP leads to adopt many methods to obtain the optimal solution of CSP. Some of them are Column Generation, SPRINT, and other heuristic methods. These methods are used to each study by situation. In specific, the methods which could improve computation time of obtaining the optimal solution or obtain the more optimized solution were selected.

To apply methods to solve CSP, other various algorithms including their own developed algorithms are adopted. Most of algorithms are used to obtain an integer value of the solution, such as Neighborhood Search algorithm, Carmen algorithm, Branch-and- 
bound algorithm. On the contrary, development of computer science leads to reducing in computational time. Software which is used to represent the algorithm of the models improved by the time, and the performance of the computer has been developing rapidly. Thus, now it is possible to obtain the optimal solution of the problem however the models are complex. However, there are problems in adopting previous models to other regions which have different situations. Because the real-world instances are used to develop the models and to evaluate, the models have a defect which cannot be adopted to different situations.

The other problem is that the previous models did not consider the importance of the quality in schedules. The problem of the quality in crew schedule, such as reducing crew's fatigue, is also being risen (Valdes and Andres, 2010). The quality of schedules leads to the quality of the service, so its importance is obvious. However, the main idea of current studies for crew scheduling is to minimize operation cost by optimizing crew schedule or to reduce the computational time for obtaining the optimal solution. Though this area is not discussed well yet, its importance is gradually increasing in recent studies. By reviewing the previous works, the implications will be suggested and the main issue for the future CSP will be discussed.

This paper is composed as follows: Section 2 describes the generalities of crew scheduling problem. Methods and algorithms to solve such methods, and models are introduced. From these, problem in present approaching methodology of CSP will be suggested. In Section 3, the summary of this paper and the main issue for the future CSP will be presented.

\section{Problem Description}

CSP is generally divided into two sub-problems: Crew pairing and Crew assignment problems. This means that CSP consists of two parts, generating pairings and assigning crews to them. In some studies, the importance of generating pairings of good quality were expressed. Because the pairings which are the output of crew pairing step become the input of crew assignment step, it is necessary to generate pairings of good quality. Though the pairings of good quality do not always guarantee the final solution of good quality, but it is impossible to obtain final solution of good quality with poor pairings (Özdemir, 2009).

In addition, CSP has the large number of trips in general. By assembling each trip, schedules for crews are created. However, the number of combinations is too many, makes the problem hard to be solved (Byun, et al., 2002). Even if the problem is solved, the optimal solution would not be obtained or the time is spent too much for computation.

On the other hand, there is various regulations required by each nation, organization or company. Typical constraints contain maximum workload without resting, minimum resting time, maximum total working time (Groot and Huisman, 2004). Without these typical constraint, many other diverse constraints are added to the problem. As a result, CSP has many constraints which make it hard to obtain the optimal solution. Many constraints make the formulation of CSP more complex (Paek, 1992). These two problems, a size of CSP and complexity of the formulation, make the problem hard to be solved and spent much time. Thus, various studies trying to reduce a size of the problem or the number of constraints have been conducted.

\subsection{Terminology Definitions}

Before reviewing models, methods, and algorithms of CSP, a few definitions of terminologies should be given. In fact, terminologies related with crew scheduling is different between countries or organizations. Also, each study defines terminologies in various way. So, widely accepted definitions will be presented here. 
1. Service: In the dictionary, it means "something that the public needs, such as transport, communications facilities, hospitals or energy supplies, which is provided in a planned and organized way by the government or an official body". In this paper, it is a little bit like this. It is the total route a bus or a subway, etc., runs from the first station to the last station.

2. Leg (or Trip, Segment): A piece of service which represents a minimum service unit. This is defined as a single non-stop run in general. Drivers may change their vehicle or take a break in the end of the leg. Passengers may get on or off the vehicle (These are not accepted in airlines. In that case, only the first definition, a piece of service, will be applied).

3. Duty (or Shift): In some studies, this is defined as a set of some legs. It is mostly a daily workload of a crew. In other studies, this may be the same with the term "Pairing". This can be confusing, but if defined clearly, it doesn't matter. Whatever the term used, it means a daily workload. Using the term "Shift" would be meaning of a set of pairings which means just a sequence of legs. By this, "Shift" means a several days of work.

4. Pairing: This can be defined as a set of some legs. In some studies, this is just a sequence of legs and means a daily workload, but in others it means a longterm workload such as a set of duties. When generating pairings, some regulations required by the nation or the transport company should be satisfied.

5. Roster: A sequence of shifts that lasts for a few weeks. It lasts for a month, but it could be changed. A crew assigned to the roster will serve different routes by shifts which consist a roster for the roster lasts.

6. Depot: This means a starting and ending base of a crew's service. Every crew has their own depot and should return to there. In case of an airline, a crew does not need to return.

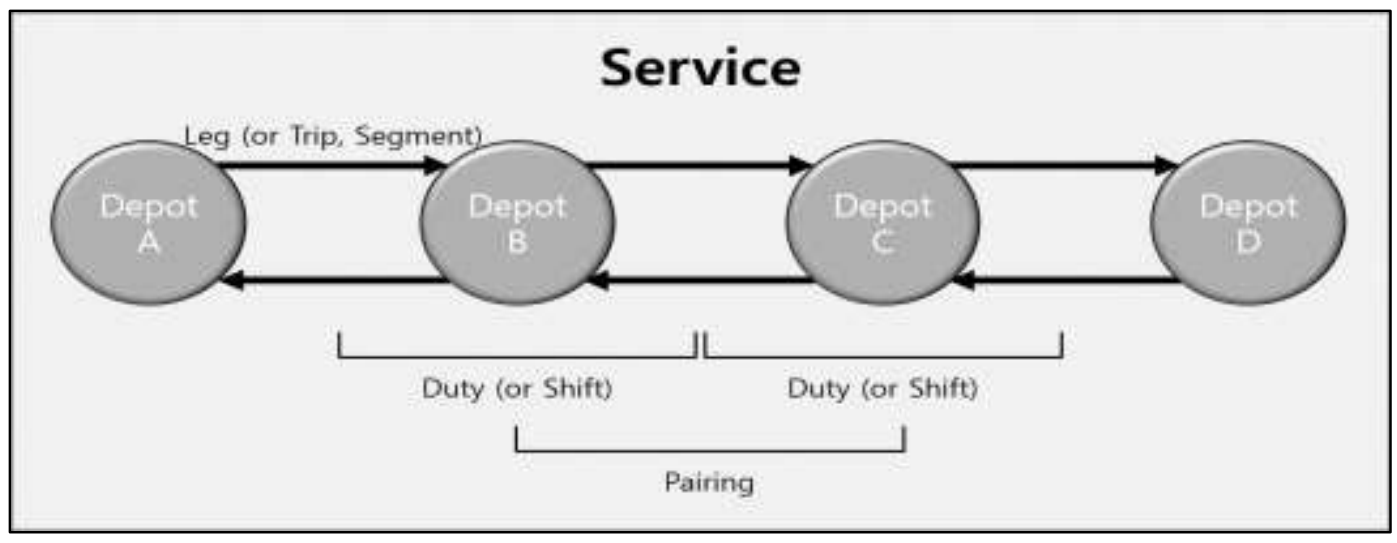

Figure 1. Graphical Representation of the Definitions

Table 1. Example of a Roster

\begin{tabular}{|c|c|c|c|c|c|c|c|c|c|c|c|c|c|c|c|c|c|c|c|c|c|c|c|c|}
\hline Day & $\mathbf{1}$ & 2 & $\mathbf{3}$ & $\mathbf{4}$ & $\mathbf{5}$ & $\mathbf{6}$ & $\mathbf{7}$ & $\mathbf{8}$ & $\mathbf{9}$ & $\mathbf{1 0}$ & $\mathbf{1 1}$ & $\mathbf{1 2}$ & $\mathbf{1 3}$ & $\mathbf{1 4}$ & $\mathbf{1 5}$ & $\mathbf{1 6}$ & $\mathbf{1 7}$ & $\mathbf{1 8}$ & $\mathbf{1 9}$ & $\mathbf{2 0}$ & $\mathbf{2 1}$ & $\mathbf{2 2}$ & 23 & 24 \\
\hline Shift X & A & A & A & A & A & A & B & B & B & B & B & B & C & C & C & C & C & C & D & D & D & D & D & D \\
\hline Shift Y & C & D & D & D & D & D & D & A & A & A & A & A & A & B & B & B & B & B & B & C & C & C & C & C \\
\hline Shift Z & B & B & B & C & C & C & C & C & C & D & D & D & D & D & D & A & A & A & A & A & A & B & B & B \\
\hline Rest & D & C & C & B & B & B & A & D & D & C & C & C & B & A & A & D & D & D & C & B & B & A & A & A \\
\hline
\end{tabular}


The graphical representation of the definitions is presented in Figure 1. Components of transportation service which are defined above are presented. 3. Duty and 4. Pairing are defined different by each study and could bring confusing situation, so it should be defined clearly. An example of a roster is presented in Table 1. As explaining above, a roster consists of some shifts. In each day, there are crews which run their shift. For this example, each shift lasts for 6 days and rest for one, two, three-day respectively. With these definitions, various studies will be reviewed. If necessary, a clear definition will also be proposed.

\subsection{Modeling of Crew Scheduling Problem}

Crew scheduling problem is expressed in Integer Programming (IP) problem, which is relaxed to Linear Programming (LP) problem to reduce its complexity and solve it. After solving LP problem, finding integer value from the solution is necessary. Such methods are various for each study. From now on, several models to express CSP will be presented.

Generally, CSP is modelled to Set Covering problem with other additional constraints. Traditional Set Covering problem is mathematically formulated as follows:

$$
\begin{aligned}
& \min \sum_{j \in J} c_{j} x_{j} \\
& \text { s.t. } \sum_{j \in J} a_{i j} x_{j} \geq 1, \quad i \in I \\
& x_{j} \in\{0,1\}, \quad j \in J
\end{aligned}
$$

Where

$I$ : a set of legs

$J$ : a set of duties

$c_{j}:$ a cost of serving duty $j$

$x_{j}$ : binary decision variable that indicates duty $j$ is belong to the solution or not.

$a_{i j}$ : coefficient that is 1 if leg $i$ is covered by duty $j$, otherwise, is 0 .

In this case, the term "Duty" or "Pairing" could be used if they mean a set of legs and a daily workload. Set Covering problem is the problem of covering the rows of $I$-row, $J$ column, zero-one matrix $\left(\mathrm{a}_{\mathrm{ij}}\right)$ by a subset of the columns at minimum cost (Beasley, 1987). Set Covering problem has been used to model CSP in various situations. Above formulation indicates that minimizing the cost of duties which are contained in the solution. The object function represents minimizing the cost. The first constraint expresses that all duties belonged to the solution must be covered. The second constraint indicates that Set Covering model is an integer programming problem, which has only binary value, 0 or 1 .

In some studies, CSP is modelled to Set Partitioning model, whose first constraint is changed as follows:

s.t. $\sum_{j \in J} a_{i j} x_{j}=1, \quad i \in I$

By replacing the inequality in the first constraint to the equality, Set Covering problem is changed to Set Partitioning problem. 
In other studies, the problem is represented as Bin Packing problem. Bin Packing problem is well-known grouping problem whose items of various size must be grouped into fixed capacity of bins (Cabral, et al., 2000). Laurent and Hao (2008) used Greedy Random Adaptive Search Procedure (GRASP), which is similar to the Best Fit Decreasing Strategy. The Best Fit Decreasing Strategy was developed for the Bin Packing problem. Through GRASP, the initial schedule which contains a feasible crew schedule and a vehicle schedule that satisfies constraints was found. Cabral, et al., (2000) developed a heuristic approach to solve Rio-Sul Airline scheduling problem using Bin Packing problem. In the study, the size of bins was set to different by crews.

Basically, modelled form of CSP needs to consider many other constraints. Adding other constraints to the model, CSP is finally modelled. The reason why these constraints should be included is to reflect real-world. In addition, it is important in the context of the efficiency in the crew assignment phase. If generating adequate sequences of trips is not considered in crew pairing phase, removing inadequate sequences of trips process is required. If proper constraints are considered, unnecessary process could be removed. Similarly, it is impossible to obtain the final solution of good quality with poor pairings. Finally, these constraints make the model to CSP model.

Constraints include the regulation required by a diversity of organizations. The maximum continuous driving time without resting, the maximum number of daily workloads to a crew, the minimum resting time, and returning to the depot after daily work are considered constraints most of studies. Oketch (2013) considered reducing the number of trip sequences (i.e., A-B-A-C-A to A-B-C-A) constraint. By reducing unnecessary routes, the problem became more concise and more easy to handle. Setting maximum duty time by each type of crew was also considered. Complying with Collective Bargain Agreements (CBA), a minimum and maximum number of hours that load to a crew in a day was considered. It is suggested that the pilots are regulated maximum 12 hours and cabin crew maximum 15 hours. Groot and Huisman (2004) assumed returning of vehicles to the depot if there is an enough time to return. The number of constraints is reduced by this assumption. Laurent and Hao (2008) considered simultaneous vehicle and crew scheduling, and various sort of vehicles. Constraints were considered separately by each problem, vehicle scheduling and crew scheduling. In vehicle scheduling phase, enough time to move from one trip-end depot to the other tripstart depot was considered. In crew assigning phase, allowing to change their vehicle or not that changes depending on the rules of companies was considered. These constraints make the model reflect real-world. Paek (1992) considered time constraints that make infeasible duties to feasible, by breaking them and removing legs that make the duty infeasible. Insurance constraints that prevent entering the algorithm and obtaining infeasible solution were also considered. This study obtained the optimal solution of CSP with the minimum number of constraints. Valdes and Andres (2010) proposed quality of the crew schedule by suggesting a variety of constraints about resting. The mechanism how to calculate days of resting was also suggested. Byun, et al., (2002) considered the constraint about a night schedule. In addition, by separating crew pairing and crew scheduling problem, constraints for generating pairings were adopted in sub-problem that represents crew pairing problem. Kim, et al., (2011) suggested the constraint that indicates the number of waiting. It is not included in the present working condition and do not influence other constraints, but a slight change of range in the constraint directly influences the number of feasible pairings. This study reduced a complexity of crew pairing by considering the constraint about the number of waiting. Borndörfer, et al., (2006) considered rule verification oracle that determines accepting generated pairings or not, to avoid too much modification in adopting constraint.

Since the number of the variables follows the number of legs, the size of the problem becomes large. It occurs because the number of possible combinations of legs exponentially increases by the number of trips increases (Byun, et al., 2002). Even 
notwithstanding this problem, the size of CSP becomes large for reflecting real-world to the model. These two things, a large size of the problem and complexity of the formulation make CSP hard to solve. Thus, studies have been conducting to find methods of solving CSP despite a large size of the problem or a large amount of constraints.

\subsection{Methodologies}

Adding other constraints to Set Covering model, the basic mathematical formulation is completed. Now the problem is an Integer Programming problem and a NP-hard, which is hard to solve. Thus, other methods are required to solve the problem. In many studies, CSP is divided into the Master problem (MP) and the Sub problem. The most popular method to solve the problem is Column Generation method. The examples of adopting Column Generation methods to solve CSP are Yunes, et al., (2000), Groot and Huisman (2004), Byun, et al., (2002), Borndörfer, et al., (2006) etc. In Column Generation method, the MP is solved with a new variable that is created through the solution of the Sub problem. First the MP is solved, and the solution of the MP is applied to solve the Sub problem. The solution of the Sub problem is added again to the MP and then the MP is solved. This procedure iterates until the object value of the MP reduces no more. If the object value of the MP does not reduce anymore, it is considered that the solution is optimal. The advantage of using Column Generation method is that not all of variables are considered at once. Only the variables that have possibility of reducing the object value of CSP will be considered gradually.

Because Column Generation method based on the linear programming, there is a limitation that the object function and the constraints of Set Covering problem must be represented to linear formulation. Thus, Set Covering problem is needed to be relaxed to linear relaxation by removing the integer constraint of CSP. For relaxing Set Covering problem, there are several studies which utilized Lagrangian relaxation such as Groot and Huisman (2004), Huisman, et al., (2005), and Borndörfer, et al., (2006). In some studies, the MP is changed to the Restricted Master Problem (RMP) and then solved. In RMP, only the restricted number of trips is considered. Combined with Column Generation method, it helps to solve the large size of the problem.

There are other methods used to solve CSP, excepting Column Generation method. SPRINT method is one example of them, which solves the problem with the subset of entire generated pairings. Özdemir (2009) used this approach. In SPRINT method, the only pairings help to improve the solution are selected after obtaining an initial solution with the subset of pairings. Other remaining pairings are added and the problem is solved with this set. This procedure is iteratively going on until there are no more pairings to add. The solution obtained from SPRINT method has the defect that does not always guarantee the optimal solution of the linear-relaxed CSP (Byun, et al., 2002).

Another method to solve the large size of the problem is dividing the large number of the trips into small subsets. Because the large number of the trips makes it harder to solve the problem, it could be appropriate approach. Groot and Huisman (2004) and Huisman, et al., (2005) split a large size of the Multi-Depot Vehicle and Crew Scheduling Problem (MD-VCSP) into the Single-Depot Vehicle and Crew Scheduling Problem (SD-VCSP). In these studies, this methodology performed well and has a low computation time. Cabral, et al., (2000) adopted the pilot-by-pilot decomposing because of difficulty in obtaining the global optimal solution. The general large problem was partitioned to smaller ones.

The method which considering crew schedule in terms of fairness is also exists. Cabral, et al., (2000) considered finding a balanced distribution of pairings by each crew. Because an unbalanced distribution of pairings was obtained through their methodology, redistribution was processed. Valdes and Andres (2010) considered the fairness in generated rosters. Using Tabu Search Procedure, the neighborhood, which means moving the shifts between the rosters was created. After creating the neighborhood, balanced average monthly working hours of the changed roster were obtained by calculating. 
On the other hand, the importance of considering crew's fatigue is not suggested in many studies. Valdes and Andres (2010) proposed an importance in regularity of crew schedule, with suggestion of how to calculate resting days of crew. The service quality of public transportation comes from crew's performance who operate their vehicle. Though the regulations exist by each nation, inappropriate schedules may cause accidents or service quality deterioration and will lead to deficit. This is why crew's fatigue must be considered. Such a thing should be treated well in the future.

\subsection{Algorithms}

Algorithms are used to find integer solution after solving linear relaxation of CSP and are various by studies. Most of them are represented as heuristic algorithms, their own developed, which are hard to classify by types. Others used simplex method to solve the linear relaxation, which is composed by relaxing integer constraints of Integer Programming to linear constraints. Some well-known used algorithms are Neighborhood Search Algorithm (Laurent and Hao, 2008; Valdes and Andres; 2010, Hwang, 2009), Carmen algorithm (Özdemir, 2009; Wedelin, 1995), Branch-and-bound algorithm (Borndörfer, 2006; Özdemir, 2009).

In Neighborhood algorithms, the solution is iteratively improved based on the optimal solution. Hill-Climbing Search, Simulated Annealing, Tabu Search are some examples which are included in Neighborhood Search (Hwang, 2009). Hill-Climbing Search starts with the random solution which is neighborhood of the current solution, and searches for improved solution by incrementally changing the input. The searching steps perform iteratively until the solution is not improved. However, there is the problem that the final solution could be the local solution, not assuring the global solution.

Simulated Annealing is similar to Hill-Climbing Search, but the input solution is changed by the probability. The probability is as follows:

$$
P\left(E\left(s^{\prime}\right), E(s), T\right)=e^{-\left(E\left(s^{\prime}\right)-E(s) / T\right)}
$$

$\mathrm{E}(\mathrm{s})$ and $\mathrm{E}\left(\mathrm{s}^{\prime}\right)$ mean the energy of the current state $\mathrm{s}$ and the new state $\mathrm{s}^{\prime}$, respectively. The substitution of $\mathrm{E}(\mathrm{s})$ and $\mathrm{E}\left(\mathrm{s}^{\prime}\right)$ means the degree of improvement in solution, and directly influences the probability of movement. T means the temperature of first state. At first, neighbor value of the solution is selected and the movement probability is calculated. Though the new state $s^{\prime}$ is worse than the current state $s$, the movement is processed. However, if the new state $s^{\prime}$ has a lot of distance with the current state $s$, the probability of movement is low. When the value of $\mathrm{T}$ is high, in the early phase, the probability of movement is high, but as the temperature being lower, the probability of movement being low. By the temperature value being 0, the optimal solution is obtained (Hwang, 2009).

Tabu Search is another neighborhood search algorithm which prohibits stuck in the local solution. In Tabu Search, the movement worsening the current solution is accepted when there is no more improvement in the solution. In addition, the movement for searching the optimal solution is not the one-way. Instead of, Tabu Search accepts a free search with some constraints. For example, the movement is only accepted when it is not a "Tabu", which prevents moving back to the local solution. In other words, Tabu Search spends more time to search for higher probability of the global solution, but less time for the local solution. Tabu Search could be an improved searching method of Hill-Climbing Search.

In Carmen algorithm, the formulation of CSP is reformulated to unconstrained, nonlinear problem by using Lagrangian relaxation. To find integer value, this algorithm does not use the solution of the LP problem, but dual values and the reduced cost. For obtaining the unique integer solution, an approximation which guarantees the solution of Lagrangian relaxation having only the value of 0 or 1 was introduced (Özdemir, 2009; 
Wedelin, 1995). This algorithm was used in CARMEN system which solves CSP of airlines such as SAS, Lufthansa, Alitalia and KLM.

Branch-and-bound is another algorithm which is used for obtaining the optimal solution. It is used for various optimization problem, especially in discrete and combinational optimization. By finding upper and lower bound rapidly, it minimizes enumeration of all feasible solutions and the computational time. In detail, the original problem is divided into several regions which could cover the original region. This process is called branching and the original problem is called root problem. Branched regions become sub-problems and then they are solved. If the optimal solution of subproblems exists, it becomes the optimal solution of the root problem, but not necessarily the global solution. For the large size of problems, such as CSP, it is hard to solve all the branched regions. Thus, the step called bounding is needed. If the solution of the subproblem exceeds the lower or the upper bound, obtained solution is proved not the global solution. These steps, branching and bounding step are used to obtain the global optimal solution of the root problem.

Algorithms were developed by commercial software, such as CPLEX, ILOG Solver, VC++, Matlab, XPRESS-MP, FORTRAN, etc. This software helps expressing their algorithms to computer language and obtaining the optimal solution. As the time passes, used version of software was also changed, in the 2000 the CPLEX 3.0 was used and in the 2010 the CPLEX 10. The performance of equipment for computation was also improved. These improvements make it possible to handle the larger size of the problem.

On the other hand, the fact that the most studies are conducted with real-world instances, which is limited to some regions, it occurs the problems when adopting their algorithms to other regions or situations. In short, it could be said there is the problem of generalization when adopting algorithms to other situations. There are several causes of this problem, one is because the regulations are different by nations or organizations. In addition, the number and the types of the trips are different by each situation. It makes hard to select the specific algorithm when adopt to other regions because the constraints need to be changed, and the model should be modified. However, constructing the general model which could be adopted in all situation is practically impossible. If it is possible to construct the general model of CSP, it could be more efficient in time because it is unnecessary to develop the model by the situation.

\section{Conclusion}

In this study, several examples of modelled CSP, its solving methodologies and algorithms are presented. CSP is modelled to Set Covering problem, Set Partitioning problem, Bin Packing problem, or the other problems by situation of each study. Excepting basic constraints included in these models, other constraints are needed to reflect the real-world. In addition, there are the numerous number of trip units. The size of the problem and the number of constraints makes the problem complex. Thus, the problem is NP-hard, which is hard to solve in most situation.

Many studies have been conducted to solve these problem, suggesting their methodologies of approaching to the optimal solution. The most popular method is Column Generation method, which iteratively uses the solution of the Sub Problem for obtaining the optimal solution of the Master Problem. When adopting Column Generation method, several studies used Lagrangian relaxation or considered the only restricted number of variables. Because of the large size of the problem, there also existed dividing the large instances into the small subsets and then solving. Some studies considered the fairness between each crew schedule. However, most studies only concentrated on reducing the object value, which represented as the operation cost or the number of used vehicles and crews. These may cause the deterioration in the quality of service, also lead 
to the deficit of the system. The generated schedule quality issue such as considering crew's fatigue arises in this context.

After solving linear relaxation, it is needed to apply the procedure for finding the integer solution from the obtained linear solution. Various algorithms are adopted in this step, such as Neighborhood Search algorithm, Carmen algorithm, Branch-and-bound algorithm etc. These algorithms are developed by using the commercial software. Improving software and computer performance help obtaining the optimal solution. Thus, it is possible to handle the problem of the large size. On the other hand, the generalization problem appeared because the former studies were mostly conducted with real-world instances and were fitted to their situation. Adopting their models, methodologies, or algorithms to more general situation is practically impossible. Thus, there is a necessity of developing models which could be applied in general situation.

This study reviewed the previous works of solving Crew Scheduling Problem. The more efficient methods to solve the problem is now being developed. Most of them are focused on minimizing operation cost by optimizing crew schedule. In numerous case, the number of crews needed for maintaining the service is calculated by fitting just to the minimum value of the regulations. This kind of studies have been conducted from old times. More important obvious things which should be considered in solving crew scheduling are exist. From now on, it would be necessary to consider the suggested problem above, not only the efficient. The effect of considering crew's fatigue has to be came out into the open and this would be treated in the future work. Accumulated fatigue caused by an inadequate crew schedule necessarily occurs the fatal defect of the system. In severe case, it could occur fatal accidents and trigger huge social losses.

In addition, having generality is also the other important thing which the future models should have. No amount of studies would improve the total system to be optimized. By the development of commercial software and the performance of computers, it is possible to handle more complex models. If the model in the previous used to solve only limited area, now it is time to develop more general models which could be adopted to solve various situation of crew scheduling problem. It means reduction of unnecessary researches and costs, and also needs a various and vast researches. For next researchers, it really is expected to research for the advanced ideas.

\section{Acknowledgement}

This work was supported by the National Research Foundation of Korea (NRF) grant funded by the Korean government (MSIP: Ministry of Science, ICT \& Future Planning) (No. NRF-2015R1A2A2A04005646).

\section{References}

[1] H. J. Ryu and V. Gosselin, "A General Framework for Manpower/Crew Scheduling Problems: The curve fitting problem revisited", Journal of the Korean Institute of Industrial Engineers., vol. 26, no. 4, (1997), pp. 402-410.

[2] C. A. Oketch, "Algorithm optimization for solving crew scheduling problems", Master's thesis, Universitat Oberta de Catalunya, (2013).

[3] T. H. Yunes, A. V. Moura and C. C. De Souza, "Solving very large crew scheduling problems to optimality", Proceedings of the 2000 ACM symposium on Applied computing-Volume 1, Como, Italy, (2000), March 19-21.

[4] S. W. de Groot and D. Huisman, "Vehicle and crew scheduling: solving large real-world instances with an integrated approach", No. EI 2004-13, (2004).

[5] B. Laurent and J. K. Hao, "Simultaneous vehicle and crew scheduling for extra urban transports", International Conference on Industrial, Engineering and Other Applications of Applied Intelligent Systems, (2008), pp. 466-475.

[6] U. Özdemir, "Methodology for Crew-Pairing Problem in Airline Crew Scheduling", PhD thesis, Boğaziçi University, (2009). 
[7] L. D. A. F. Cabral, M. J. Freitas, N. Maculan and R. C. V. Pontes, "An heuristic approach for large scale crew scheduling problems at Rio-Sul airlines", Proceedings 40th International Symposium of the AGIFORS, Istanbul, Turkey, (2000) August 20-25.

[8] G. H. Paek, "Iterative time constraint addition algorithm for the crew scheduling problem", Journal of the Korean Operations Research and Management Science Society, vol. 17, no. 3, (1992), pp. 159-170.

[9] V. Valdes and V. Andres, "Integrating Crew Scheduling and Rostering Problems", PhD thesis, Universitá Di Bologna, (2010).

[10] J. H. Hwang, "Integration of integer programming and neighborhood search algorithm for solving a nonlinear optimization problem", Journal of The Korea Society of Computer and Information, vol. 14, no. 2, (2009), pp. 27-35.

[11] J. I. Byun, K. S. Lee, S. S. Park and S. Y. Kang, "An Integer Programming Approach to the Subway Daily Crew Scheduling Problem", Journal of the Korean Operations Research and Management Science Society, vol. 27, no. 4, (2002), pp. 67-86.

[12] T. S. Kim, K. Y. Park, T. J. Park and K. R. Ryu, "Candidate Dia Creation Methods for Subway Crew Scheduling", Proceedings of the Korean Information Science Society Conference, vol. 38, no. 1, (2011).

[13] J. I. Byun, K. S. Lee and S. S. Park, "An Integer Programming Approach to the Problem of Daily Crew Scheduling", Proceedings of the Korean Operations Research and Management Science Society, (2000), pp. 613-616.

[14] J. H. Hwang, C. H. Park, Y. H. Lee and K. R. Ryu, "Crew Schedule Optimization by Integrating Integer Programming and Heuristic Search", Journal of KIISE: Computing Practices and Letters, vol. 8, no. 2, (2002), pp. 195-205.

[15] D. Huisman, R. Freling and A. P. Wagelmans, "Multiple-depot integrated vehicle and crew scheduling", Transportation Science, vol. 39, no. 4, (2005), pp. 491-502.

[16] R. Borndörfer, U. Schelten, T. Schlechte, and S. A. Weider, "A column generation approach to airline crew scheduling”, Operations Research Proceedings 2005, (2006), pp. 343-348.

[17] J. E. Beasley, “An algorithm for set covering problem”, European Journal of Operation Reseach, vol. 31, no. 1, (1987), pp. 85-93.

[18] D. Wedelin, "An algorithm for large scale 0-1 integer programming with application to airline crew scheduling", Annals of operations research, vol. 57, no.1, (1995), pp. 283-301. 